\title{
Performance evaluation and operation of Auto Load transfer switch
}

\author{
Diponkar Paul, Md. Shariful Islam, Syed Khalid Rahman, Md. Saiful Islam, Mir Mohiuddin
}

World University of Bangladesh, Dhaka, Bangladesh

Email address:

DIPO0001@e.ntu.edu.sg (D. Paul), islam_eee@yahoo.com (M. S. Islam), oberon.syed@gmail.com (S. K. Rahman), saiful_ete07ruet@yahoo.com (M. S. Islam),munshi12@yahoo.com (M. Mohiuddin)

\section{To cite this article:}

Diponkar Paul, Md. Shariful Islam, Syed Khalid Rahman, Md. Saiful Islam, Mir Mohiuddin. Performance Evaluation and Operation of Auto Load Transfer Switch. International Journal of Renewable and Sustainable Energy. Vol. 2, No. 6, 2013, pp. $205-211$.

doi: $10.11648 /$ j.ijrse.20130206.13

\begin{abstract}
An automatic transfer switch is an integral component of an emergency power supply system (EPSS). Auto transfer switch (ATS) consists of different electrical and mechanical components such as magnetic contactor, relay, timer, over under voltage relay etc. Basically maintenance includes scheduled and unscheduled renovation works. Efficiency of auto transfer switch (ATS) can be increased by using proper operation \& standard maintenance schedule. Maintenance procedures are normally used to reduce unexpected problems of auto transfer switch. Proper installation, commissioning, operation, maintenance \& troubleshooting procedure have been discussed thoroughly in this project report. A large number of real pictures have been shown to make the report more useful to all. It has been tried to provide a good understanding of the ideas about proper operation, maintenance \& troubleshooting of auto transfer switch. Some auto transfer switches allow load shedding prioritization of optional circuits, such as heating and cooling equipment. More complex emergency switchgear used in large backup generator installations permits soft loading, allowing load to be smoothly transferred from the utility to the synchronized generators, and back; such installations are useful for reducing peak load demand from a utility. In cases where two independent utility feeders are available, a fast load transfer can be achieved by the use of a static transfer switch. When the critical load includes a load transformer, the transformer is subjected to transients during the transfer interval. Therefore following a load transfer, a transient current flows into the transformer which can cause a post transfer voltage sag. Fault disturbance characteristics which determines voltage difference between the sources and the voltage drop across t he incoming and the outgoing thyristor switches during the load transfer process.
\end{abstract}

Keywords: ATS, Earthing Bar, AC \& DC Timer Base

\section{Introduction}

The application of automatic switches for transferring load, consideration should be given to the relative costs of air break switches versus oil circuit breakers. This is especially true when the circuit or circuits under consideration, at some future date, might become the source for important load centers. It is not the intent of the authors to discuss the costs nor to outline the relative advantages of one type of switching scheme over another. It might be pointed out, however, that automatic air break can be installed readily in such a manner that, when the circuit conditions warrant, oil circuit breakers can be added and the original air break switches used for by-pass switching. The principal function of an automatic load transfer scheme is to transfer a load from a preferred source, upon failure of voltage on the preferred source, upon failure of voltage on the preferred line, to an emergency source, thus restoring service to the station. The second function is to transfer the load back to the preferred source upon restoration of voltage on the preferred line. This latter transfer should be made without interruption to service which necessitates paralleling the two lines for a short time during the transfer. The principal function of an automatic line sectionalizing and load transfer scheme is to first sectionalize the line by opening both line switches upon failure of voltage on the bus, followed by the closing of one of the switches when voltage is restored on one section of the line, thus restoring service to the station. If desired, normal service can be restored by additional provisions in the control scheme for closing the second line switch when voltage is restored on its section of the line. Due to lack of 
proper knowledge for standard manufacturing and maintenance of ATS such as ATS electrical drawing, design, assembly, electrical connection, improper safety protection, maintenance schedule we are facing several problems after manufacturing of the ATS. But ATS is now one of the most important part for our electricity transmission sector. If we know and consent to maintain standard manufacturing design and maintenance procedure of ATS manufacturing and maintenance works then we will come out from those problems easily. For the sequence our Internship title is Manufacturing of Automatic Transfer Switch. Designing and manufacturing an ATS following different standards [1] Study on different components those are used in ATS to select appropriate materials, equipments and to ensure proper installation and troubleshooting. Knowing about standard manufacturing process. To understand the assembly procedure of ATS. To identify different types of trouble and necessary measures related with ATS. Suggest possible solution of the identified problem. This report is also have some limitations such like we cannot get the soft copy of datum from the computers of Company for having confidential. So, we lost some data. We also get very little chance to pick the photos because that is a restricted and sensitive area. They don't allow us to carry cell phones and camera. That why we can gather some photos only from non- restricted part of the company.A transfer switch is an electrical switch that reconnects electric power source from its primary source to a standby source. Switches may be manually or automatically operated. An Automatic Transfer Switch (ATS) is often installed where a backup generator is located, so that the generator may provide temporary electrical power if the utility source fails.

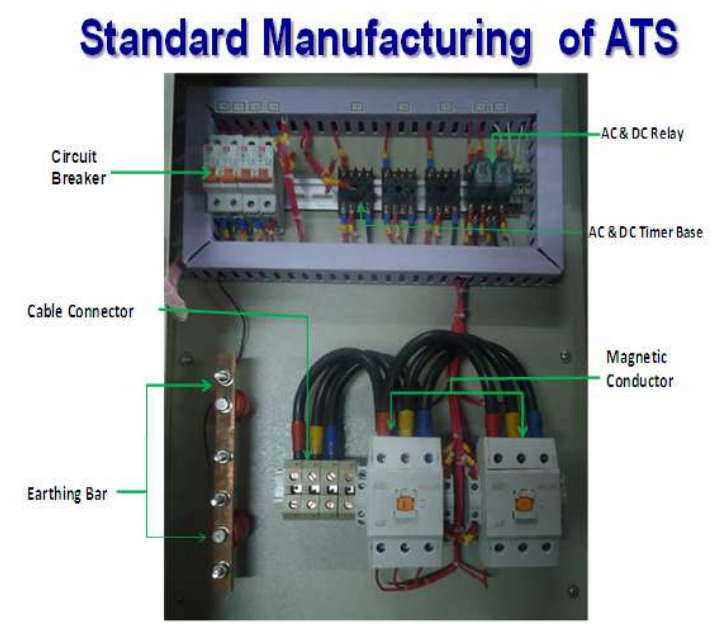

Figure 1: Standard Manufacturing of Automatic Transfer Switch

As well as transferring the load to the backup generator, an ATS also commands the backup generator to start, based on the voltage monitored on the primary supply. The transfer switch isolates the backup generator from the electric utility, when the generator is on and is providing temporary power. The control capability of a transfer switch may be manual only, or a combination of automatic and manual. The switch transition mode of a transfer switch may be Open Transition (OT) (the usual type), or Closed Transition (CT). For example, in a home equipped with a backup generator and an ATS, when an electric utility outage occurs, the ATS will tell the backup generator to start. Once the ATS sees that the generator is ready to provide electric power, the ATS breaks the home's connection to the electric utility and connects the generator to the home's main electrical panel. The generator supplies power to the home's electric load, but is not connected to the electric utility. Isolation of the generator from the distribution system is required to protect the generator from overload, and to prevent accidental energization of the service wiring. When utility power returns for a set time, the transfer switch will transfer back to utility power and command the generator to turn off, after another specified amount of "cool down" time with no load on the generator. A transfer switch can be set up to provide power to only critical circuits or entire electrical (sub) panels [2]. Some transfer switches allow for load shedding or prioritization of optional circuits, such as heating and cooling equipment. More complex emergency switchgear used in large backup generator installations permits soft loading, allowing load to be smoothly transferred from the utility to the synchronized generators, and back; such installations are useful for reducing peak load demand from a utility. An open transition transfer switch is also called a break before make transfer switch. A break before make transfer switch breaks contact with one source of power before it makes contact with another. It prevents backfeeding from an emergency generator back into the utility line, for example. One example is an open transition automatic transfer switch (ATS). During the split second of the power transfer the flow of electricity is interrupted. Another example is a manual three position circuit breaker, with utility power on one side, the generator on the other, and "off" in the middle, which requires the user to switch through the full disconnect "off" position before making the next connection. A closed transition transfer switch is also called a make before break transfer switch. In a typical emergency system, there is an inherent momentary interruption of power to the load when it is transferred from one available source to another (keeping in mind that the transfer may be occurring for reasons other than a total loss of power). In most cases this outage is inconsequential, particularly if it is less than 1/6 of a second. There are some loads, however, that are affected by even the slightest loss of power. There are also operational conditions where it may be desirable to transfer loads with zero interruption of power when conditions permit. For these applications, closed transition transfer switches can be provided. The switch will operate in a make-before-break mode provided both sources are acceptable and synchronized. Typical parameters determining synchronization are: voltage difference less than $5 \%$, frequency difference less than $0.2 \mathrm{~Hz}$, and relative phase angle between the sources of 5 electrical degrees. Since the maximum frequency difference is $0.2 \mathrm{~Hz}$, the 
engine will generally be required to be controlled by an isochronous governor. It is generally required that the closed transition, or overlap time, be less than 100 milliseconds. If either source is not present or not acceptable (such as when normal power fails) the switch must operate in a break-before-make mode (standard open transition operation) to ensure no back feeding occurs. Closed transition transfer makes code-mandated monthly testing less objectionable because it eliminates the interruption to critical loads [3], which occur during traditional open transition transfer. With closed transition transfer, the on-site engine generator set is momentarily connected in parallel with the utility source. This requires getting approval from the local utility company. A softloading transfer switch actively changes the amount of load accepted by the generator. A static transfer switch uses power semiconductors such as Silicon-controlled rectifiers (SCRs) to transfer a load between two sources. Because there are no mechanical moving parts, the transfer can be completed rapidly, perhaps within a quarter-cycle of the power frequency. Static transfer switches can be used where a reliable and independent second source of power is available and it is necessary to protect the load from even a few power frequency cycles interruption time, or from any surges or sags in the prime power source.

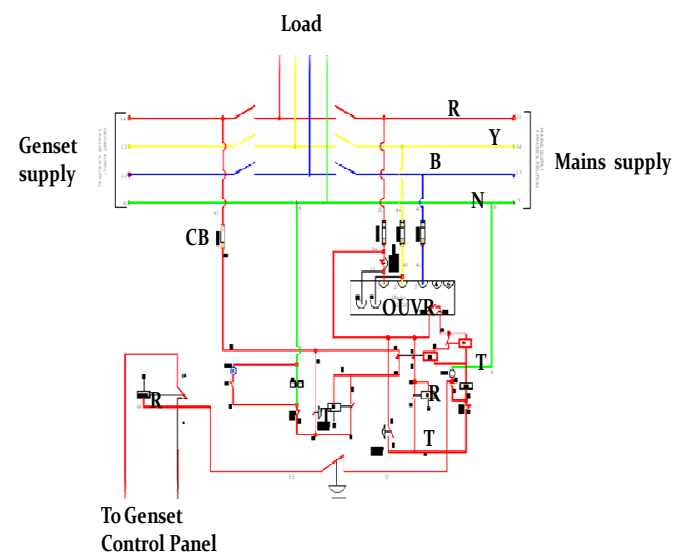

Figure 2: Typical load switching application for closed transmission

Typical load switching applications for which closed transition transfer is desirable include data processing and electronic loads, certain motor and transformer loads, load curtailment systems, or anywhere load interruptions of even the shortest duration are objectionable. A closed transition transfer switch (CTTS) is not a substitute for a UPS (uninterruptible power supply); a UPS has a built-in stored energy that provides power for a prescribed period of time in the event of a power failure. A CTTS by itself simply assures there will be no momentary loss of power when the load is transferred from one live power source to another. Homes with standby generators may use a transfer switch for a few circuits or the whole home. Different models are available, with both manual and automatic transfer. Often small transfer switch systems use circuit breakers with an external operating linkage as the switching mechanism. The linkage operates two circuit breakers in tandem, closing one while opening the other. Manufacturers of transfer switches can provide installation guides to select the size of switch and provide recommended installation procedures. Like all other electrical apparatus, local electrical codes require transfer switches to carry safety approvals. However, some transfer switches are sold via the Internet, and there have been problems with counterfeit circuit breakers. The magnetic contactor electricity passes in through one contact and into the contactor's parent device in this manner [6]. To deactivate, the contactor can be physically pulled from the parent device. Also, in the absence of electrical current, the spring pushes the core away from the coil, breaking the connection. Magnetic contactors are a form of electrical relay found on most electrically powered motors. They act as a go-between for direct power sources, and high-load electrical motors in order to homogenize or balance out changes in electrical frequency which may come from a power supply as well as to act as a safeguard. It should be noted that though they are similar in design, magnetic contactors are not circuit breakers. They do not sever the connection between appliance, and power source during a short circuit. They are detachable from a motor so that an operator may work with that motor; disassemble or maintain it, without the possibility of live current still passing through the device. When electricity flows through the magnetic contactor, it causes the electromagnet to generate a strong magnetic field. This field pulls the iron core into the coil, and creates an electrical arc. Electricity passes in through one contact and into the contactor's parent device in this manner. To deactivate, the contactor can be physically pulled from the parent device. Also, in the absence of electrical current, the spring pushes the core away from the coil, breaking the connection. A magnetic contactor has three parts: power contacts, contact springs and auxiliary contacts. The power contact creates, carries and breaks the current in a magnectic contactor. The contact springs create a sufficient amount of pressure on the contacts. Auxiliary contacts perform signaling and interlocking maneuvers. Contactors vary in size and capacity. A contactor may be small enough to fit in your hand or it may be several feet on one side. AVAILABILITY of reliable semiconductor switches and stringent voltage quality requirements of sensitive loads have made mediumvoltage thyristor-based Static transfer Switches (STSs) attractive substitutes to Electro-Mechanical Transfer Switches (EMTSs) during the past few years. A STS can provide fast transfer of a sensitive load to an alternate source of ac power when the preferred source fails [3]-[5]. Performance evaluation of a STS, which is primarily determined by the transfer time, is not trivial due to the involvement of various system parameters and component characteristics. The transfer time is of significant importance in evaluation of a STS performance since it identifies duration of power discontinuity/interruption for 
the sensitive load. Duration of power discontinuity is the key factor in predicting proper operation of the load. A STS must be able to perform a fast load transfer from the disturbed source to the healthy one regardless of the load type and the fault/disturbance characteristics. Power discontinuity, as a result of load transfer process by means of a STS, is determined by the total load-transfer time $\left(t_{\text {tot }}\right)$ The total load-transfer time is the sum of 1) detection time ( $t_{\text {det }}$ - delay in voltage detection logic) and 2) transfer time ( $t_{t r}$-delay in load transfer mechanism). Performance of a STS, with respect to transfer time, under various fault/disturbance scenarios is investigated in [1] when the sensitive load is represented by a passive RL load. This paper evaluates a STS performance for hybrid ( $\mathrm{RL}+$ motor) loads. It describes the effects of a regenerative (motor) load on the STS transfer time and explains the relation between fault/disturbance characteristics and regenerative mode of operation of the hybrid load. It also identifies the worst case scenario(s) in which the maximum transfer time can occur. Commutation process in each phase is determined by the following two factors: 1) Voltage drop across the alternate source incoming thyristor switch after the fault/disturbance detection. 2) Direction of the current flow in the corresponding phase. Parameters which affect the commutation process between the thyristor switches, and thus the transfer time, are listed in [1]. These parameters are: Thyristor gating method which is the strategy of removing gating signals from $\mathrm{Ti}$ thyristor switches and triggering $\mathrm{T} 2$ thyristor switches. Load type, parameters and characteristics which determine the relative positions of phase voltage and line current zero-crossings and the voltage drop across the incoming and the outgoing thyristor switches. Fault/disturbance characteristics which determine voltage difference between the sources and the voltage drop across the incoming and the outgoing thyristor switches during the load transfer process.

\section{Methodology}

Station L normally is served from station OC which in turn is served from a 69,000-volt transmission line connecting stations OR and P. Oil circuit breakers 488 at station OR and 588 at station P normally are closed and are reclosed manually after automatic tripping. Oil circuit breakers 177 at station OC and 188 at station OR normally are closed with control arranged for one immediate reclosure after automatic tripping and two time delay reclosures. The total reclosing cycle is approximately $2^{1} / \mathrm{t}$ minutes. At station $\mathrm{L}$ motor operated air break switch 152 normally is closed and 252 normally open with their controls arranged for automatic switching. AUTOMATIC SWITCHING FOR FAULT ConDitions. Failure of Line Potential. Failure of voltage on the incoming line from station OC causes, after a time delay of approximately three minutes, the opening of switch 152 followed by the closing of switch 252 . This switching is initiated by the de-energized contact of auxiliary line voltage relay UV-X which is normally energized through energized contracts of line voltage relays UV-1 and UV-2. Return of Line Potential. Return of potential on the line from station OC causes, after a short time delay, switch 152 to close, which for a few seconds parallels the two sources, followed by the opening of switch 252 , thus restoring normal service. This operation is initiated by the energized contacts of relays UV-1 and UV-2 and is interlocked with synchronism check relay SCR. Failure of voltage on the incoming line from station OC, which caused the original switching operation, might have resulted from the tripping of breaker 177 at station OC or from the tripping of breakers 488 and 588 at stations OR and P. The instructions for switching on the 69,000 - volt line after a trip-out call for first closing breaker 488 at station OR followed by synchronizing and closing breaker 588 at station P. Because of the possibility of the reverse switching operations, that is, closing switch 588 before 488 , thus energizing station $\mathrm{L}$ from a different generating source, and in order to serve stations $\mathrm{OC}$ and $\mathrm{L}$ from station $\mathrm{P}$ in an emergency with switch 488 open at station OR, the automatic control at station $\mathrm{L}$ is provided with a synchronism check relay. This relay blocks an attempt to parallel the two sources during this switching operation unless they are in synchronism. One coil of the synchronism check relay is connected to the bus potential transformer and the other to one phase of the line potential transformers. Failure of Bus Potential Following Line Potential Failure. Failure of voltage on the incoming line from station OR after switching operation 1 has taken place causes no further switching. If this conditions still exists when voltage is restored on the line from station OC, switch 252 will open and 152 will close. This operation is initiated by auxiliary line voltage relay UV-X and bus voltage relay UV-3. Failure or Restoration of Potential during Switching Operations. In the foregoing three switching operations, it is assumed that the initiating voltage conditions continue until a switching operation is completed. In switching operation 1 , if line potential is restored on the line from station $\mathrm{OC}$ before relay TD completes its timing cycle, no switching will take place. If line potential is restored after TD contact closes but before 152 -fr contact is closed, switch 152 will open, 252 remains open and 152 will re- close by the operation of relay CX$1 \mathrm{~A}$ as in switching operation 3 . In switching operation 2 , if voltage again fails on the incoming line from station OC before the contact of the synchronism check relay SCR closes, no switching takes place. If voltage fails on this line after relay CX-1 has closed its contacts, switch 152 will close and 252 will open. Under this condition switching operation 1 will be repeated. In switching operation 3, if voltage fails on the incoming line from station $\mathrm{OC}$ after relay TX-2A contact closes but before 252 -a closes, switch 252 will open but will reclose by the operation of relay CX2 as in switching operation 1 . When the critical load includes a load transformer [Fig. 1(b)], the transformer is subjected to transients during the transfer interval. 
Therefore, following a load transfer, a transient current flows into the transformer which can cause a post-transfer voltage sag. It is important to design the STS control system to distinguish a post-transfer voltage sag from those due to the feeder disturbances. A post-transfer voltage sag is a function of the transformer parameters (e.g., saturation characteristics, the line impedances, and the transfer process). The transient flux is determined by (1) the load transformer parameters, (2) fault/disturbance characteristics, (3) the time needed for the detection of a disturbance in the system (i.e., detection time), and (4) the time needed for the load transfer (i.e., transfer time). A fast STS was introduced in [4]. The STS employs (1) a voltage detection logic based on transforming ac voltages into a synchronously rotating frame and (2) a selective gating strategy based on the direction of current flow. Performance of the voltage detection logic is reported in [5]. The transfer scheme performance is also evaluated in [6]. In [5] and [6], various disturbances are considered, and the corresponding detection time/transfer time curves are derived. Two utility feeders, one of which is the preferred source and the other one is the alternate source; sensitive load; STS which can connect the load to either of the sources; control system which monitors the quality of the sources voltages, and performs a load transfer if needed. To estimate the transient flux of a load transformer during a transfer process, the single-phase equivalent circuit diagram of a three-phase Delta/Wye transformer is used. Fig. 4 depicts this equivalent circuit. Precise calculation of the transient flux is not trivial due to the complexity of the STS behavior and nonlinearity of the load transformer. To make the analytical expressions manageable, the following simplifying assumptions are made: 1) thyristor switches are ideal; 2) load transformer is linear; 3) utility feeders are in-phase; 4) line impedances are neglected to estimate the peak transient flux; 5) no paralleling occurs between the two feeders. Under normal operating conditions, the transformer is connected to the preferred source. Therefore, if the line impedances are neglected, then $v_{t}=v_{p}=v_{a}=v_{p} \cos (\omega t+\phi) \quad$ where $\mathrm{v}_{\mathrm{t}}$ is the transformer input voltage, $v_{p}$ is the preferred- source voltage, $v_{a}$ is the alternate-source voltage, $v_{p}$ is the peak voltage, and ( $\mathrm{p}$ is the phase angle. As shown, the power source is given to the electromagnet through a control switch and through contacts to the load. When current starts flowing through the control coil, the electromagnet starts energizing and thus intensifies the magnetic field. Thus the upper contact arm starts to be attracted to the lower fixed arm and thus closes the contacts causing a short circuit for the power to the load. On the other hand, if the relay was already de-energized when the contacts were closed, then the contact move oppositely and make an open circuit. As soon as the coil current is off, the movable armature will be returned by a force back to its initial position. This force will be almost equal to half the strength of the magnetic force. This force is mainly provided by two factors. They are the spring and also gravity. Relays are mainly made for two basic operations. One is low voltage application and the other is high voltage. For low voltage applications, more preference will be given to reduce the noise of the whole circuit. For high voltage applications, they are mainly designed to reduce a phenomenon called arcing. The Transfer switch isolates selected circuits from the home with "Break" then "Make" switches. Each switch has 3-positions; LINE, OFF and GEN (generator). In the LINE position, utility power feeds the selected circuits. In the GEN position, generator power feeds the selected circuits. To switch from LINE to GEN, the switch is passed through the OFF position. This allows a positive break to occur, which prevents sparking, arcing or short circuits that might be created if the positive break was not part of the system. Individual circuit protectors protect each selected circuit from overload. This allows larger generator to be connected to the transfer switch without the transfer switch being overloaded This test is only possible in automatic mode, it allows to start the genset even if the mains is unavailable before and during a critical period on the mains. This test simulates a loss of mains condition. Loss of mains sequence is started and main's return sequence automatically activated as soon as generator is available All timers are run following their setting. The DBT is running not only during the "main's return" sequence but during the "loss of main's" sequence tool. Test on load inhibits return to zero position feature on the main and genset side to limit power shut down ("RETURN TO 0" make at NO in the two sides). The "retransfer inhibit" feature is always actived during test on load (from keypad) Press "TEST" pushbutton to make test on load Led blinking, press "validation" pushbutton to enter the code and start the test cycle, following loss of main + main's return sequences. After the test, the automatic mode is active. It is possible to remotely start the "test on load" closing the contact 207 and 209 on the control terminal. The cycle is started as soon as the input is closed. Theretransfer from generator to main is blocked, and only allowed once the input is de-activated. Return to zero feature unavailability. Unavailability of this optional feature will be indicated on the front panel. Please refer to the trouble shooting guide. Return to zero position feature is realised thanks to a internal energy storage device [2]. In case of failure of this component, an alarm message will be displayed. This alarm must reset via validation pushbutton and a pointer remains on to inform operator that return to zero feature ("2nd TRIP") is no longer operational. Troubleshooting is the process or identifying and fixing problems. It is used to fix problems with hardware, and many other products. The basic theory of troubleshooting is starting with the most general (and often most obvious) possible problems, and then narrow it down to more specific issue. 


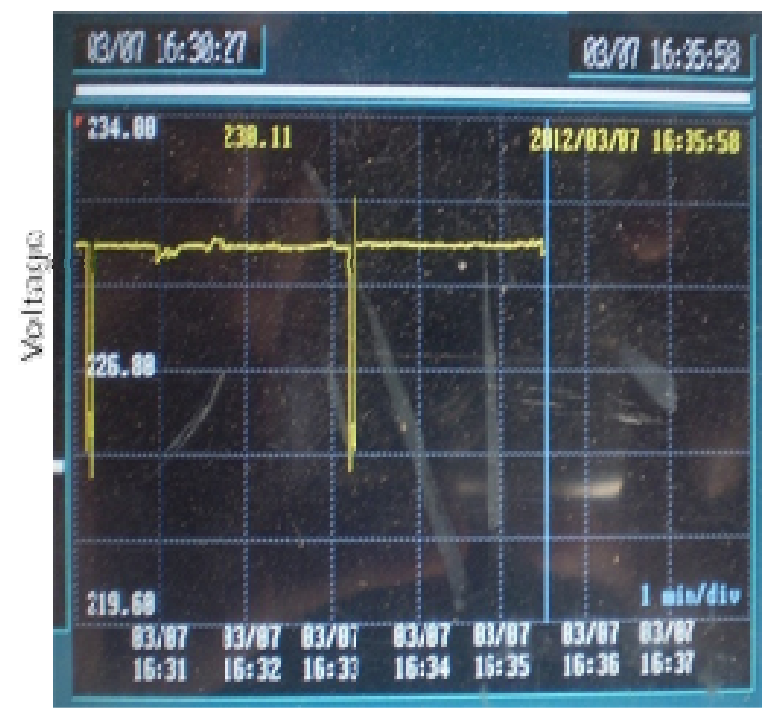

Figure 3: Time versus voltage curve with changing of load demand by voltage control scheme

When load increases, voltage temporarily decreases and for the very short time, voltage offset Created. Auto load transfer switch by voltage control scheme, makes operation stable. Upon loss of power the automatic transfer type station will always transfer itself to its second available source of power provided that second source is energized. The automatic sectionalizing type station will always pick itself up upon whichever line become energized and will close the sections together if both become energized. If a fault occurs on, the bus between the automatic air switches of either transfer type or sectionalizing type, both air switches will, after unsuccessful attempt to re-energize the bus, open and remain open until manual switching is performed. With both switches thus locked open it is evident that all section of the line can be energized from their respective source of power. Our method operation differs in an outstanding point from that of Rich and Kirwen. There is no need of co-ordination of timing between the operation of the breakers and the feed points in the operation of the automatic air switches, except that the breaker should not close until the air break switches have opened [5], that is, the first re closure should not be less than about three seconds. We do not delay the operation of our air-switches until tests are made by the automatic breakers at the source stations. Instead, the transfer type installation at once transfers its load to the second source; the sectionalizing type at once opens both of its airswitches. The action in both cases is initiated by an under voltage time delay relay set to close its contacts in one second at zero volts. The load of the transfer type installation is thus re-energized within three seconds; the load of the sectionalizing installation is energized after an interruption not exceeding about nine seconds. If both line sections become energized, the two air-switches will close restoring the entire line to service in the case of the sectionalizing type of install.

\section{Conclusion}

The experimental measured waveforms for a single phase to ground fault are shown as voltage offset drawing a very short time. The fault occurs at $t 1=10.3 \mathrm{~ms}$ in phase a of the STD-2 benchmark model. It is detected by the voltage detection logic at $t 2=15.85$. Therefore, commutation does not begin until the alternate source incoming switch is forward biased. Commutation begins at the zero crossing of phase a voltage which occurs at $\mathrm{t} 3=20.8 \mathrm{~ms}$ resulting in a transfer time of $4.95 \mathrm{~ms}$. The total load transfer time is $10.5 \mathrm{~ms}$. For a given system, the peak transient flux is a function of detection and transfer time intervals. The shorter the total load transfer time, the less severe is the transient flux and the transient current. Detection and transfer time intervals themselves are also functions of the load type and the disturbance characteristics. The worst case condition with respect to the peak transient flux may occur for loads that can become regenerative. For an $\mathrm{RL}+$ motor load, depending on the disturbance scenario [5], the commutation can fail and the maximum transfer time may occur. Such a case can result in the maximum peak transient flux. The post-transfer voltage sag in the system may cause the STS to mistakenly disconnect the load from the healthy source. Therefore, the STS control logic must operate such that no power interruption would occur after the transfer is completed, provided that the alternate source is in a better condition than the preferred one. This Paper reports performance of a STS system in terms of 1) Transfer Time and 2) Total transfer time when its hybrid sensitive load is composed of a RL load and a motor load. It is assumed that the STS under investigation operate based on a selective gating strategy. The Paper concludes that: A hybrid load composed of RL and motor load can operate in a regenerative mode during the load transfer process depending on the motor and the RL load parameters [4]. .For a hybrid load with specific RL and motor load parameters, fault/disturbance characteristics e.g., type of fault and the fault instant, determine if the load becomes regenerative during transfer process. The maximum transfer is determined by the phase in which commutation fails and the load becomes regenerative. If a load becomes regenerative, the transfer time is determined by the zero crossing instants of the system voltage, i.e., phase voltage or line voltage zero crossing. In this paper, the transient behavior of a load transformer during a sub cycle bus transfer is investigated. Due to the time intervals required for the detection of a disturbance and transferring the load, the load transformer will be subjected to a transient flux. As a result, a transient current flows into the transformer which causes a post-transfer voltage sag [6]. The thyristor switches of the STS must be designed such that they can tolerate the transient current within a transfer. This control scheme includes the general requirements previously outlined for automatic load transfer schemes. There also is provided a synchronism check interlock to block an attempt to parallel the two sources during 
switching operations unless they are in synchronism. In the design of automatic air break switch control schemes, the first consideration is the potential source to use for operation and control. Some schemes require a source of potential independent of the lines being switched while others can be operated with duplicate sources. One connected to each of the two main transmission lines. Another important consideration is the completion of a transfer, once it is started or to set up the control so that a transfer will take place when one of the lines is energized. The automatic switching schemes described are for single phase control only- that is, one potential transformer for each line. The use of two potential transformers for ungrounded system or three for grounded systems should be given consideration. Single phase control has been chosen and failure to operate and consequent outage has resulted when line wires have been broken. Additional potential transformers and relay are to then be installed to prevent such failures.

\section{References}

[1] F. W. Rich, M. S. Kirwen, (1947) "Automatic control of air switches for line sectionalizing and load transfer, "AIEE transactions, vol\# 66, ppno \#293-302

[2] Hossein Mokhtari, Sashi B. Dewan, (2001)," Effect of regenerative load on a static transfer switch performance "IEEE transactions on power delivery, vol\# 6, No\# 4.

[3] H. Mokhtari, M. R. Iravani, S. B. Dewan, “ Bench mark systems for digital computer simulation of a static transfers switch, "IEEE transactions on power delivery submitted for publication.

[4] H. Mokhtari, "High speed silicon controlled rectifier static transfer switch/ "in IEEE $30^{\mathrm{TH}}$ Annual meeting.

[5] H. Mokhtari, M. R. Iravani, S. B. Dewan, (2003), " Transient behavior of load transfer during sybcycle bus transfer, IEEE transactions on power delivery, vol\# 18, No\# 4.

[6] H. Mokhtari, S. B. Dewan, M. R. Iravani, (2000), "performance evaluation of thyristor based static transfer switch, "IEEE transactions on power delivery, vol\# 15, pp no\# 960-966. 